\title{
IDIOPATHIC PORTAL HYPERTENSION OCCURRING IN A SYSTEMIC SCLEROSIS PATIENT: CASE REPORT
}

\author{
Ana Cecília de Sena Oliveira ${ }^{1}$, João Victor de Pinho Costa ${ }^{1}$, Caio Carvalhais Chaves ${ }^{1}$, Débora Cerqueira Calderaro ${ }^{1, \star}$ \\ 1.Universidade Federal de Minas Gerais, Belo Horizonte (MG), Brazil. \\ *Corresponding author: dccalderaro@gmail.com
}

\section{BACKGROUND}

The overlap of idiopathic portal hypertension (IPH) and systemic sclerosis (SSc) is rare, and case reports of its occurrence are scarce in the literature. This case describes the case of a patient with SSc developing IPH.

\section{CASE REPORT}

A 52-year-old woman, diagnosed with diffuse SSc in 2015, started treatment prednisolone 60 mg + monthly intravenous (IV) pulse therapy with cyclophosphamide (CYC). In the third month of treatment, she developed a scleroderma renal crisis, and, therefore, prednisolone was rapidly tapered and $150 \mathrm{mg} /$ day of captopril and hemodialysis were initiated. Monthly IV CYC was performed for 6 months. Hemodialysis was interrupted after completing 18 months. Prednisolone was suspended in 2016. Maintenance treatment with mycophenolate or azathioprine were not tolerated. Since 2017 she has not been on immunosuppressive treatment for SSc. Systemic sclerosis cutaneous involvement improved, chronic kidney disease is stable and no additional systemic complication of SSc developed. In 2019, she was hospitalized to investigate ascites. Ascites was dealt with diuretics. During hospitalization, portal hypertension with small and medium esophageal varices, in addition to collateral circulation were diagnosed. From 2019 to 2020, hepatic diseases were evaluated through extensive propaedeutic. Tomography of the abdomen and pelvis showed ascites. Transaminases and canalicular enzymes, coagulation and albumin remained normal. Serology for viral, schistosomiasis mansoni and autoimmune hepatitis were negative. Abdominal Doppler ultrasound depicted increased portal vein diameter and patent umbilical vein with retrograde flow (presence of portosystemic shunt) and splenomegaly. Magnetic resonance cholangiopancreatography was unremarkable. Hepatic elastography depicted normal liver stiffness and the absence of steatosis. In 2021, a liver biopsy was performed, showing mildly active interface hepatitis, without fibrosis or steatosis. The final conclusion was SSc associated IPH. Currently, she is using captopril $300 \mathrm{mg} /$ day, clonidine $0.4 \mathrm{mg} /$ day, allopurinol $300 \mathrm{mg} /$ day, carvedilol $50 \mathrm{mg} / \mathrm{day}$ and minoxidil $7 \mathrm{mg} /$ day and omeprazole $20 \mathrm{mg} /$ day. The only medication addressing portal hypertension is carvedilol, prescribed for primary prophylaxis of esophageal varices bleeding. Recurrent ascites treated with intermittent diuretics rarely occur.

\section{CONCLUSION}

Idiopathic portal hypertension is a disorder of unknown etiology, characterized by a hepatic venous gradient higher than normal range. Systemic sclerosis is an autoimmune disease characterized by microvascular damage, generalized fibrosis in different organs and dysregulation of innate immunity. Although further studies are necessary to shed light on both SSc and IPH pathogenesis, evidence of shared mechanisms of vascular fibrosis may be a useful source of hypothesis to the understanding of possible shared identity and/or causal relationship between them.

\section{KEYWORDS}

Systemic sclerosis, Idiopathic portal hypertension, Doppler ultrasound, Transient elastography, Liver biopsy. 